\title{
The activity of echinocandins, amphotericin B and voriconazole against fluconazole-susceptible and fluconazole-resistant Brazilian Candida glabrata isolates
}

\author{
Débora Alves Nunes Mario ${ }^{1,2} /{ }^{+}$, Laura Bedin Denardi', Laíssa Arévalo Bandeira', \\ Milene Silva Antunes ${ }^{3}$, Janio Morais Santurio', Luiz Carlos Severo3 ${ }^{3}$, Sydney Hartz Alves ${ }^{1,2}$
}

'Departamento de Microbiologia e Parasitologia ²Programa de Pós-graduação em Ciências Farmacêuticas, Centro de Ciências da Saúde, Universidade Federal de Santa Maria, Santa Maria, RS, Brasil ${ }^{3}$ Complexo Hospitalar Santa Casa de Misericórdia, Porto Alegre, RS, Brasil

The extensive use of azole antifungal agents has promoted the resistance of Candida spp to these drugs. Candida glabrata is a problematic yeast because it presents a high degree of primary or secondary resistance to fluconazole. In Brazil, C. glabrata has been less studied than other species. In this paper, we compared the activity of three major classes of antifungal agents (azoles, echinocandins and polyenes) against fluconazole-susceptible (FS) and fluconazole-resistant (FR) C. glabrata strains. Cross-resistance between fluconazole and voriconazole was remarkable. Among the antifungal agents, the echinocandins were the most effective against FS and FR C. glabrata and micafungin showed the lowest minimal inhibitory concentrations.

Key words: Candida glabrata - echinocandins - antifungal drug resistance

In Europe and the United States of America (USA), Candida glabrata has emerged as the second most common cause of invasive candidiasis and an increasing number of reports show its importance in mucosal or bloodstream infections (Pfaller \& Diekema 2007). Systemic infections due to C. glabrata are characterized by a high mortality rate and they are difficult to treat due to their intrinsically low susceptibility to azoles, particularly fluconazole (Pfaller et al. 2004). Numerous C. glabrata isolates have shown primary resistance to fluconazole, while others easily develop fluconazole resistance after exposure to the treatment (Bennett et al. 2004).

In Brazil, Colombo et al. (2006) evaluated the aetiology of candidaemia episodes in 11 Brazilian medical centres and showed that they occur in 2.49 cases for every 1,000 hospital admissions, which is two-15 times more frequently than those reported for centres in the Northern Hemisphere. However, based on percentages (4.9\%), C. glabrata is apparently less important in Brazil (it holds 5th place among the Candida species), but it corresponds to 0.12 cases for every 1,000 hospital admissions, which is similar to Europe and the USA.

Echinocandins are antifungal agents licensed for the treatment of invasive candidiasis by the USA Food and Drug Administration (Brielmaier et al. 2008). These agents disrupt the cell walls of Candida species by inhibiting $\beta-1.3-\mathrm{D}$-glucan synthase, which results in cell rupture and death (Messer et al. 2009). Echinocandins

Financial support: CAPES

+Corresponding author: debora_alves6@hotmail.com

Received 25 July 2011

Accepted 25 October 2012 prevent the overlapping toxicities and drug interactions with mammalian cells observed between azoles and polyenes (Wiederhold \& Lewis 2003).

In 2000, caspofungin was the first echinocandin to be marketed in Brazil. In 2009, anidulafungin was introduced in Brazil as a treatment for invasive candidiasis in non-neutropenic adult patients. Recently, in 2010, micafungin was approved by the National Agency of Sanitary Surveillance for use in Brazil and designated for the treatment of invasive candidiasis, prophylaxis in individuals undergoing haematopoietic stem cell transplant, Aspergillus infections and empiric therapy for febrile neutropenia in adults and children, as previously established in Europe and Japan. Micafungin has been studied in both the USA and Brazil; however, it is currently only approved in the USA for use in adults (Bormann \& Morrison 2009).

The aim of this study was to evaluate the in vitro activity of anidulafungin, micafungin and caspofungin against fluconazole-susceptible (FS) and fluconazoleresistant (FR) C. glabrata clinical strains that were isolated from candidaemia episodes in Brazil and compare them to amphotericin B and voriconazole.

Anidulafungin and voriconazole (Pfizer, Inc, New York, NY), amphotericin B and fluconazole (Sigma Chemical Co, St. Louis, MO), caspofungin (Merck Research Laboratories, Rahway, NJ) and micafungin (Fujisawa Pharmaceutical Co Ltd, Osaka, Japan) were obtained as standard powders and prepared according to the Clinical and Laboratory Standards Institute guidelines (CLSI 2008a, b).

We studied two groups of $C$. glabrata strains. The first group included clinical FS isolates $(\mathrm{n}=36)$ obtained from blood cultures and from deep-site specimens or oropharyngeal exudates. The second group included FR $(\mathrm{n}=36)$ derivatives obtained from susceptible isolates through an in vitro method of fluconazole resistance in- 
duction described by Fekete-Forgács et al. (1999). All of the yeasts were tested by the broth microdilution method using the CLSI M27-A3 and third informational supplement (M27-S3/2008) standardized reference method.

The Mann-Whitney U test was used to compare two independent samples to evaluate the different groups (susceptible strains vs. resistant strains).

The results of the susceptibility test against the systemically active antifungal agents for the two C. glabra$t a$ strain groups are presented in Tables I and II.

All of the isolates that were subcultured with fluconazole according Fekete-Forgács method acquired resistance to fluconazole; the minimal inhibitory concentrations (MICs) to fluconazole in the FR group ranged from $64.0-256.0 \mu \mathrm{g} / \mathrm{mL}$ and the differences between the MICs of both groups were significant $(\mathrm{p}<0.0001)$ (Table II).

Based on susceptibility parameters (MIC range, $\mathrm{MIC}_{50}, \mathrm{MIC}_{90}$ and geometric mean), the FS C. glabrata group was very susceptible to amphotericin $\mathrm{B}$, showing a $\mathrm{MIC}_{90}$ of $0.125 \mu \mathrm{g} / \mathrm{mL}$ and a geometric mean of $0.093 \mu \mathrm{g} / \mathrm{mL}$. For inhibition, the FR C. glabrata group required higher concentrations of amphotericin $\mathrm{B}$, with a $\mathrm{MIC}_{90}$ of $2 \mu \mathrm{g} / \mathrm{mL}$ and a geometric mean of $0.824 \mu \mathrm{g} /$ $\mathrm{mL}$. In this group, the MIC range included nine strains with a MIC of $2 \mu \mathrm{g} / \mathrm{mL}$. The Mann-Whitney U test

\section{TABLE I}

Susceptibility $(\mu \mathrm{g} / \mathrm{mL})$ of Candida glabrata strains to fluconazole before and after the induction of fluconazole resistance

\begin{tabular}{lcccc}
\hline $\begin{array}{l}\text { Group of isolates } \\
(\mathrm{n})\end{array}$ & Range & $\begin{array}{c}\text { Geometric } \\
\text { mean }\end{array}$ & $\begin{array}{c}\mathrm{MIC}_{50} \\
(\mu \mathrm{g} / \mathrm{mL})\end{array}$ & $\begin{array}{c}\mathrm{MIC}_{90} \\
(\mu \mathrm{g} / \mathrm{mL})\end{array}$ \\
\hline FS (36) & $1-32$ & 4.404 & 4 & 32 \\
FR (36) & $64-256$ & 140.9 & 128 & 256 \\
\hline
\end{tabular}

FR: fluconazole-resistant C. glabrata; FS: fluconazole-susceptible C. glabrata; MIC: minimal inhibitory concentration. showed significant differences between the susceptibility of FS vs. FR groups $(\mathrm{p}<0.0001)$. C. glabrata shows a natural tendency to become resistant compared with other Candida species (Pfaller \& Diekema 2004), which was confirmed in this study.

Amphotericin B is generally recommended for primary therapy of severe infections by $C$. glabrata. However, it is becoming evident that the agent is not universally effective against $C$. glabrata and higher doses of amphotericin B may be required for efficient treatment (Kremery \& Barnes 2002).

Susceptibility tests of the FS group to voriconazole showed a $\mathrm{MIC}_{90}$ of $1 \mu \mathrm{g} / \mathrm{mL}$ and a geometric mean of 0.389 $\mu \mathrm{g} / \mathrm{mL}$. On the other hand, the FR group was evidently resistant to this triazole, with a $\mathrm{MIC}_{90}$ of $16 \mu \mathrm{g} / \mathrm{mL}$ and a geometric mean of $6.229 \mu \mathrm{g} / \mathrm{mL}$. The susceptibility between the two groups was significantly different $(p<0.0001)$.

The cross-resistance of $C$. glabrata to fluconazole and voriconazole was remarkable, showing a $\mathrm{MIC}_{50}$ of $8 \mu \mathrm{g} / \mathrm{mL}$, a $\mathrm{MIC}_{90}$ of $16 \mu \mathrm{g} / \mathrm{mL}$ and a geometric mean of $6.229 \mu \mathrm{g} / \mathrm{mL}$ to voriconazole. Cross-resistance among azoles is a well-established phenomenon; therefore, fluconazole was proposed as a surrogate marker for voriconazole susceptibility, given the greater potency of voriconazole in comparison to fluconazole (Pfaller et al. 2007). Voriconazole, ravuconazole and posaconazole have shown efficacy against most FR Candida spp. These triazoles have also been significantly more effective against $C$. glabrata than fluconazole. Therefore, voriconazole has been suggested as an alternative therapy for C. glabrata strains that are resistant to fluconazole (Johnson \& Kauffman 2003). However, when comparing MICs, the correlation between voriconazole and fluconazole cross-resistance is evident (Table II) (Bennett et al. 2004). Thus, we have confirmed that azoles are less effective against $C$. glabrata, as has been shown in previous studies (Pfaller \& Diekema 2004).

The class of echinocandins showed the best activity against the FR group and the lowest MIC values were observed with micafungin. The FS group susceptibility profiles based on $\mathrm{MIC}_{90}$ were: micafungin $(0.008 \mu \mathrm{g} / \mathrm{mL})$,

TABLE II

Susceptibility $(\mu \mathrm{g} / \mathrm{mL})$ in vitro of Candida glabrata isolates antifungal agents before and after the induction of fluconazole resistance

\begin{tabular}{lccccc}
\hline Agents & Group of isolates & Geometric mean & Range & MIC $_{50}$ & MIC $_{90}$ \\
\hline Amphotericin B & FS & 0.093 & $0.062-0.250$ & 0.125 & 0.125 \\
& FR & 0.824 & $0.125-2$. & 1 & 2 \\
Voriconazole & FS & 0.389 & $0.060-2$ & 0.5 & 1 \\
Anidulafungin & FR & 6.229 & $1-128$ & 8 & 16 \\
Caspofungin & FS & 0.017 & $0.004-0.031$ & 0.004 & 0.031 \\
\multirow{2}{*}{ Micafungin } & FR & 0.031 & $0.008-0.062$ & 0.008 & 0.062 \\
& FS & 0.106 & $0.015-0.250$ & 0.125 & 0.5 \\
& FR & 0.194 & $0.062-0.50$ & 0.250 & 0.5 \\
\end{tabular}

FR: fluconazole-resistant C. glabrata; FS: fluconazole-susceptible C. glabrata; MIC: minimal inhibitory concentration. 
anidulafungin $(0.031 \mu \mathrm{g} / \mathrm{mL})$ and caspofungin $(0.5 \mu \mathrm{g} /$ $\mathrm{mL}$ ). The $\mathrm{MIC}_{90}$ required by the FR group was similar to the FS group. Based on those parameters, the C. glabrata isolates of both groups were susceptible to echinocandins. In contrast, the statistical test comparing the MICs of the FS group vs. the MICs of the FR group indicated significant differences for caspofungin $(p=0.0023)$, anidulafungin $(\mathrm{p}<0.0001)$ and micafungin $(\mathrm{p}<0.0001)$.

We have demonstrated that echinocandins exhibited potent activity against all (FS and FR) C. glabrata strains. Micafungin showed the best antifungal activity. It has also been shown to be efficient against Candida biofilms and reduce the adherence of both azole-susceptible and azole-resistant $C$. albicans strains to epithelial cells (Borg-von Zepelin et al. 2002). Echinocandins may play an important role in therapy when azole resistance emerges. In this study, we found some disparity in the MIC values among the echinocandins. Anidulafungin and micafungin have demonstrated similar activities, but caspofungin required higher doses ( $\mathrm{MIC} \pm 4$ dilutions) to inhibit strain growth (Table II). Ostrosky-Zeichner et al. (2003) found that micafungin was, on average, four times more potent than caspofungin, except when it was tested against Candida parapsilosis, which is consistent with our results. However, Perlin (2007) found that Candida spp FKS1 mutants expressing resistance to caspofungin were also cross-resistant to micafungin and anidulafungin. Pfeiffer et al. (2010) recently reported a case of $C$. glabrata breakthrough fungemia during micafungin treatment, with MIC values 4-8 $\mu \mathrm{g} /$ $\mathrm{mL}$ and FKS mutations. Such episodes emphasize the importance of monitoring azole and echinocandins resistance in C. glabrata.

Echinocandins are a class of antifungal agents with distinct mechanisms of action (Messer et al. 2009). However, the previous reports of multi-drug resistant Candida spp (Moudgal et al. 2005) involving azoles and amphotericin B or azoles and echinocandins suggest a connection between these two phenomena. Posteraro et al. (2006) reported a case of C. glabrata showing crossresistance to multiple antifungal drugs in candidaemia treatment. Our results demonstrate that the MICs of echinocandins in the FS and the FR groups have increased slightly. Acquired resistance or reduced susceptibility to echinocandins has rarely been reported, with the majority of cases being associated with mutations in the FKS1 gene (Park et al. 2005).

We must emphasize that, based on susceptibility parameters $\left(\mathrm{MIC}_{50}, \mathrm{MIC}_{90}\right.$ and $\mathrm{MIC}$ range) and established breakpoints, the FR C. glabrata group was susceptible to three echinocandins. Notably, however, when we applied a statistical test to compare the susceptibility between the two groups, the FR group was less susceptible than the FS group. Consequently, the continued application of antifungal susceptibility testing for the conventional and new antifungal agents is critical to detect the emergence of resistance in this important opportunistic fungal pathogen.

This study has clearly demonstrated the unparalleled spectrum and potency of echinocandin antifungal agents against a collection of $C$. glabrata with different suscepti- bility profiles. Micafungin, in particular, showed excellent effectiveness against the two groups of C. glabrata strains. Micafungin was the second drug in the echinocandin class to be approved in the USA and is efficient against Candida and Aspergillus spp. Overall, micafungin has proven to be safe and well tolerated and has few drug interactions. Future studies should include testing micafungin alone and as a component of combined antifungal therapy for invasive and refractory mould infections.

\section{REFERENCES}

Bennett JE, Izumikawa K, Marr KA 2004. Mechanism of increased fluconazole resistance in Candida glabrata during prophylaxis. Antimicrob Agents Chemother 48: 1773-1777.

Borg-von Zepelin M, Zaschke K, Gross U, Monod M, Müller FM 2002. Effect of micafungin (FK463) on Candida albicans adherence to epithelial cells. Chemotherapy 48: 148-153.

Bormann AM, Morrison VA 2009. Review of the pharmacology and clinical studies of micafungin. Drug Des Devel Ther 3: 295-302.

Brielmaier BD, Casabar E, Kutzeborn CM, Mckinnon PS, Ritchie DJ 2008. Early clinical experience with anidulafungin at a large tertiary care medical center. Pharmacother 28: 64-73.

CLSI - Clinical and Laboratory Standards Institute 2008a. Reference method for broth dilution antifungal susceptibility testing yeasts. Approved standard M27-A3, 3rd ed., CLSI, Wayne, 25 pp.

CLSI - Clinical and Laboratory Standards Institute 2008b. Reference method for broth dilution antifungal susceptibility testing yeasts. Approved standard M27-S3, 3rd ed., CLSI, Wayne, 19 pp.

Colombo AL, Nucci M, Park BJ, Nouer AS, Arthington-Skaggs B, Matta DA, Warnock D, Morgan J, the Brazilian Network Candidemia Study 2006. Epidemiology of candidemia in Brazil: a nationwide sentinel surveillance of candidemia in eleven medical centers. J Clin Microbiol 44: 2816-2823.

Fekete-Forgács K, Gyurc L, Lenkey B 1999. Changes of virulence factors accompanying the phenomenon of induced fluconazole resistance in Candida albicans. Mycoses 43: 273-279.

Johnson LB, Kauffman CA 2003. Voriconazole: a new triazole antifungal agent. Clin Infect Dis 36: 630-637.

Kremery V, Barnes AJ 2002. Non-albicans Candida spp causing fungemia: pathogenicity and antifungal resistance. J Hosp Infect 50: 243-260.

Messer SA, Moet GJ, Kirb JT, Jones RN 2009. Activity of contemporary antifungal agents, including the novel echinocandin anidulafungin, tested against Candida spp, Cryptococcus spp and Aspergillus spp: report from the SENTRY Antimicrobial Surveillance Program (2006 to 2007). J Clin Microbiol 47: 1942-1946.

Moudgal T, Little T, Boikov D, Vazquez JA 2005. Multiechinocandin and multiazole-resistant Candida parapsilosis isolates serially obtained during therapy for prosthetic valve endocarditis. Antimicrob Agents Chemother 49: 767-769.

Ostrosky-Zeichner L, Oude Lashof AM, Kullberg BJ, Rex JH 2003. Voriconazole salvage treatment of invasive candidiasis. Eur $J$ Clin Microbiol Infect Dis 22: 651-655.

Park S, Kelly R, Kahn JN, Robles J, Hsu MJ, Register E, Li W, Vyas V, Fan H, Abruzzo G, Flattery A, Gill C, Chrebet G, Parent SA, Kurtz M, Teppler H, Douglas CM, Perlin DS 2005. Specific substitutions in the echinocandin target FKS1p account for reduced susceptibility of rare laboratory and clinical Candida spp isolates. Antimicrob Agents Chemother 49: 3264-3273.

Perlin DS 2007. Resistance to echinocandin-class antifungal drugs. Drug Resist Update 10: 121-130. 
Pfaller MA, Diekema DJ 2004. Rare and emerging opportunistic fungal pathogens: concern for resistance beyond Candida albicans and Aspergillus fumigatus. J Clin Microbiol 42: 4419-4431.

Pfaller MA, Diekema DJ 2007. Epidemiology of invasive candidiasis: a persistent public health problem. Clin Microbiol Rev 20: 133-163.

Pfaller MA, Messer SA, Boyken L, Hollis RJ, Rice C, Tendolkar S, Diekema DJ 2004. In vitro activities of voriconazole, posaconazole and fluconazole against 4,169 clinical isolates of Candida spp and Cryptococcus neoformans collected during 2001 and 2002 in the ARTEMIS global antifungal surveillance program. Diagn Microbiol Infect Dis 48: 201-205.

Pfaller MA, Messer SA, Boyken L, Rice C, Tendolkar S, Hollis RJ, Diekema DJ 2007. Use of fluconazole as a surrogate marker to predict susceptibility and resistance to voriconazole among 13,338 clinical isolates of Candida spp tested by Clinical and Laboratory Standards Institute-recommended broth microdilution methods. J Clin Microbiol 45: 70-75.

Pfeiffer CD, Garcia-Effron G, Zaas AK, Perfect JR, Perlin SD, Alexander BD 2010. Breakthrough invasive candidiasis on micafungin. J Clin Microbiol 48: 2373-2380.

Posteraro B, Sanguinetti M, Fiori B, La Sorda M, Spanu T, Sanglard D, Fadda G 2006. Caspofungin activity against clinical isolates of azole cross-resistant Candida glabrata overexpressing efflux pump genes. J Antimicrob Chemother 58: 458-461.

Wiederhold NP, Lewis RE 2003. The echinocandin antifungals: an overview of the pharmacology, spectrum and clinical efficacy. Expert Opin Investig Drugs 12: 1313-1333. 